\title{
Current structure and flow pattern on the electron separatrix in reconnection region
}

\author{
Ruilong Guo ${ }^{1,2^{*}}\left(\mathbb{D}\right.$, Zuyin $\mathrm{Pu}^{2}$ and Yong Wei ${ }^{1}$
}

\begin{abstract}
Results from 2.5D Particle-in-cell (PIC) simulations of symmetric reconnection with negligible guide field reveal that the accessible boundary of the electrons accelerated in the magnetic reconnection region is displayed by enhanced electron nongyrotropy downstream from the $X$-line. The boundary, hereafter termed the electron separatrix, occurs at a few $d_{\mathrm{e}}$ (electron inertial length) away from the exhaust side of the magnetic separatrix. On the inflow side of the electron separatrix, the current is mainly carried by parallel accelerated electrons, served as the inflow region patch of the Hall current. The out-of-plane current density enhances at the electron separatrix. The dominating current carriers are the electrons, nongyrotropic distribution functions of which contribute significantly to the perpendicular electron velocity by increasing the electron diamagnetic drift velocity. When crossing the separatrix region where the Hall electric field is enhanced, electron velocity orientation is changed dramatically, which could be a diagnostic indicator to detect the electron separatrix. In the exhaust region, ions are the main carriers for the out-of-plane current, while the parallel current is still mainly carried by electrons. The current density peak in the separatrix region implies that a thin current sheet is formed apart from the neutral line, which can evolve to the bifurcated current sheet.
\end{abstract}

Keywords: Magnetic reconnection, Separatrix region, Electron separatrix, Diamagnetic current, Electron nongyrotropy, Finite gyroradius Effect

\section{Background}

Magnetic reconnection is a key process in space plasmas to change magnetic topology and accelerate and heat particles. The reconnection region is separated into two inflow regions and two outflow regions by four separatrices together with the $X$-line. The separatrix is a region where the field and plasma quantities are different from those of the inflow region and outflow region. The features and structures of the separatrix region at the magnetopause have been observed by Cluster (Khotyaintsev et al. 2006; Retinò et al. 2006; Lindstedt et al. 2009). These observations mainly recorded the magnetospheric branch of the separatrix region at tens of $d_{i}$ (ion inertial length) away from the $X$-line. They showed density cavity,

\footnotetext{
*Correspondence: grl@mail.iggcas.ac.cn

${ }^{1}$ Key Laboratory of Earth and Planetary Physics, Institute of Geology and Geophysics, Chinese Academy of Sciences, Beijing 100029, China Full list of author information is available at the end of the article
}

strong electric field, electron beams, intense waves, and potential jump existing in the separatrix region, suggesting that the separatrix is highly structured, especially on the low- $\beta$ side in the asymmetric case. Waves (e.g., low/ upper hybrid waves, Langmuir waves, electrostatic solitary waves, and whistler waves) are found and surveyed in separatrix region (Farrell et al. 2002; Drake et al. 2003; Viberg et al. 2013; Fujimoto 2014). In addition, temperature anisotropy has been shown to occur in both the inflow region and separatrix region in simulation results (e.g., Cattell et al. 2005; Egedal et al. 2012) and observations (e.g., Chen et al. 2009; Egedal et al. 2008, 2010, 2012). A large-scale parallel electric field is proposed to lead to this temperature anisotropy by heating the electrons in the parallel direction to form elongated electron velocity distribution functions (eVDFs) (Egedal et al. 2012), and similar eVDFs have been observed and compared with the simulation results (Egedal et al. 2010, 2012; Wang et al. 2016). The beam-type eVDF is a 
significant feature in the separatrix region as well (e.g., Cattell et al. 2005; Chen et al. 2008; Hwang et al. 2013), which can provide free energy to stimulate waves. The parallel beams have also been confirmed and studied in the magnetopause reconnection region by investigating the recent MMS (Magnetospheric Multiscale) data (e.g., Hwang et al. 2016; Phan et al. 2016). Electron nongyrotropy/agyrotropy is another important characteristic in the electron diffusion region (EDR) and separatrix region. To illustrate the nongyrotropic property of the electrons, several parameters such as electron agyrotropy $A \Phi_{e}$ (Scudder and Daughton 2008), the local degree of nongyrotropy $D_{\text {ng }}$ (Aunai et al. 2013), and Q-parameter for the measure of gyrotropy (Swisdak 2016) have been proposed. All these parameters are enhance inside and outside of EDR (e.g., Aunai et al. 2013; Shuster et al. 2015; Nakamura et al. 2016; Swisdak 2016). The nongyrotropic properties are significant in the asymmetric reconnection region, which is related to the crescent distribution generated by the finite gyroradius effect (Hesse et al. 2014; Burch et al. 2016; Hesse et al. 2016; Norgren et al. 2016; Shay et al. 2016).

The understanding of magnetic reconnection dynamics in space relies on the technique of resolving kinetic processes within the region where the plasmas are decoupled from the magnetic field. Of notable values to reconnection research are the electron-scale layers of the out-ofplane current density $\left(J_{y}\right.$, here we define $y$-axis is along the current flow direction, i.e., the out-of plane direction, while $z$-axis is perpendicular to the current sheet, and $x$-axis is along the background magnetic field) and the electric field normal to the current sheet $\left(E_{z}\right)$, as the simultaneous enhancements of these two quantities may indicate an encounter of the electron diffusion region (EDR) according to Particle-in-cell (PIC) simulation studies (Fujimoto 2006; Chen et al. 2008; Drake et al. 2008), and in situ measurements (Chen et al. 2008). Recently, several reconnection diffusion regions have been identified and investigated with the high cadence MMS measurements (e.g., Burch et al. 2016; Burch and Phan 2016; Hwang et al. 2016). Notable thin out-of-plane current sheet and out-of-plane accelerated electron flow have been recorded at dayside magnetopause to define the EDR (Burch et al. 2016; Chen et al. 2016). The generalized Ohm's law can also be studied by analyzing the MMS data, and the results showed that the divergence of electron pressure tensor significantly contributes to in-plane and reconnection electric fields (Torbert et al. 2016). The previous PIC studies showed that enhancements of $J_{y}$ and $E_{z}$ can also occur near the separatrices away from the EDR (e.g., Aunai et al. 2013; Shuster et al. 2015). In addition, Runov et al. $(2003,2006)$ found bifurcated current sheet distributions in the magnetotail by Cluster, which is suggested to be related to the magnetic reconnection. The bifurcated current structure is also reproduced by global MHD simulation by Thompson et al. (2006). The current density peak of the bifurcated current sheet does not coincide with the neutral line, and it is generally suggested that it is related to the Hall effect currents generated in separatrix region (e.g., Runov et al. 2003). The complicated current system may be also related to the reconnection front current structures (Yao et al. 2013), in which parallel current density is carried by electrons (Yao et al. 2016), and perpendicular currents are mainly carried by ions (Yao et al. 2017). These studies imply that the current system in the separatrix region is an essential element in the reconnection process.

In this paper, we present results from a PIC simulation of symmetric reconnection with zero guide field to address the formation of thin current sheet in the separatrix region and the associated electron/ion flow pattern. The finite gyroradius effect on the electron separatrix leads to electron nongyrotropy and increases the out-ofplane velocity, thereby enhancing the Hall effect. From the viewpoint of fluid, the thin current sheet on separatrix is mainly maintained by the electron diamagnetic drift.

\section{Simulation model}

The 2.5D PIC simulation in this paper uses open boundary conditions (Daughton et al. 2006, 2009). The simulation starts with an equilibrium Harris current sheet with zero guide field. The initial magnetic field is $B_{x}(z)=B_{0} \tanh (z / L)$, where $L$ denotes the halfwidth of the current sheet and $B_{0}$ is the asymptotic value of the magnetic field. The initial number density is $n(z)=n_{0} \sec h^{2}(z / L)+n_{b}$, where $n_{0}$ and $n_{b}$ represent the maximum density in the center of the Harris current sheet and the background density, respectively. The simulation domain is $L_{x} \times L_{z}=80 d_{i} \times 20 d_{i}$, containing $10,240 \times 2560$ cells with 600 particles per cell, where $d_{i}=c / \omega_{\mathrm{pi}}$ is the ion inertial length based on $n_{0}$. Other initial parameters are the mass ratio $m_{i} / m_{e}=400$, temperature ratio $T_{i} / T_{e}=5$, $L / d_{i}=0.5, n_{b} / n_{0}=0.05$, and the ratio between the electron plasma frequency and cyclotron frequency $\omega_{\text {pe }} / \omega_{\text {ce }}=2$. Reconnection is initiated by adding a perturbation $\delta B\left[\delta B_{x}=-\frac{\delta}{2}\left(\frac{L_{x}}{L_{z}}\right) \cos \left[\frac{2 \pi\left(x-0.5 L_{x}\right)}{L_{x}}\right] \sin \left(\frac{\pi z}{L_{z}}\right)\right.$, $\delta B_{z}=\delta B \sin \left[\frac{2 \pi\left(x-0.5 L_{x}\right)}{L_{x}}\right] \cos \left(\frac{\pi z}{L_{z}}\right)$, more details in Daughton et al. (2009)] to the initial magnetic field. The reconnection rate reaches its peak of $\sim 0.17$ at $t=18.5 \omega_{\mathrm{ci}}^{-1}$, where $\omega_{\mathrm{ci}}$ is the ion cyclotron frequency determined by $B_{0}$. The eVDFs are calculated in spatial bins of size $2 d_{e} \times 2 d_{e}$, where $d_{e}=c / \omega_{\mathrm{pe}}$ is the electron 
inertial length using $n_{0}$. The velocity is normalized by the ion Alfvén speed $V_{A}$ based on the initial asymptotic magnetic field $B_{0}$ and Harris sheet density $n_{0}$, yielding $V_{A}=1 / 40 c$.

\section{Simulation results and discussion}

The magnetic separatrix field lines are marked by white lines with $A_{y}=A_{y}(X)$ in Fig. $1\left(A_{y}\right.$ is the $Y$-component of magnetic vector potential, and $X$ is the coordinate of the $X$-line at $[x, z] \sim[842,0] d_{e}$ located by the saddle point of $\left.A_{y}\right)$. The data are from $t=18 \omega_{\mathrm{ci}}^{-1}$, at around the time of the peak reconnection rate. The two inflow regions are at top and bottom, while the exhaust regions (where accelerated electrons can be expelled) are at the left and right sides of the plot. At this point of time, a primary island exists

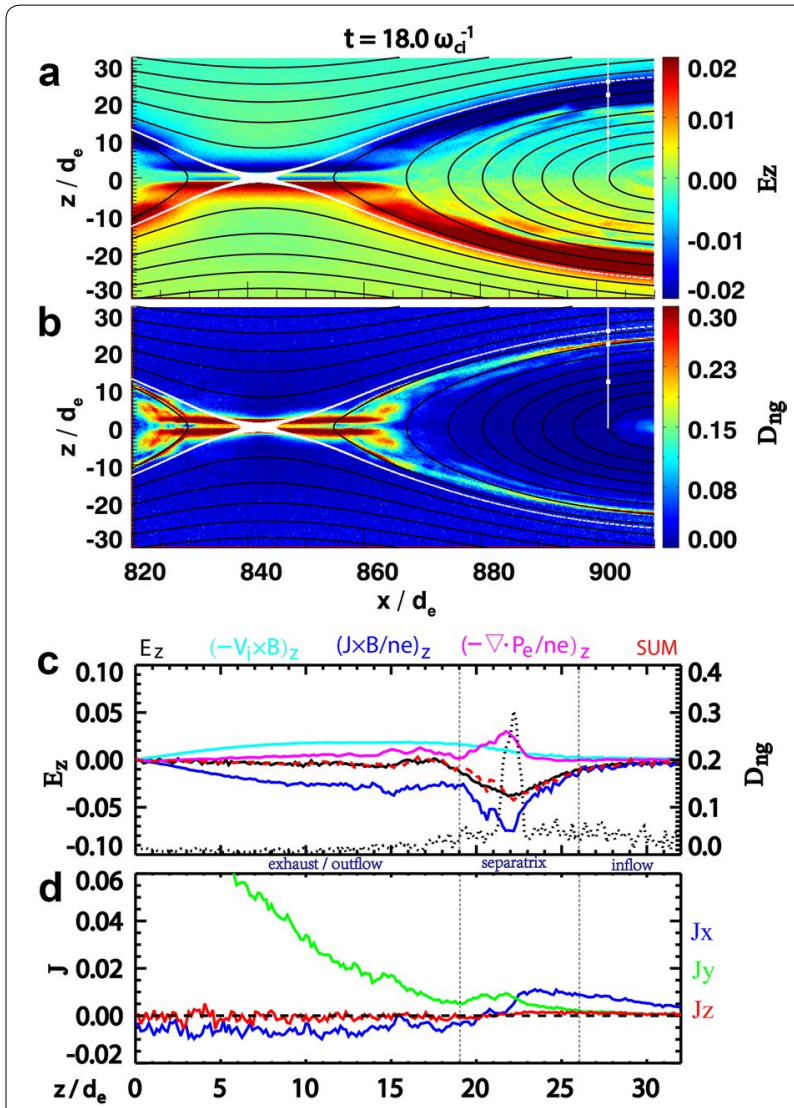

Fig. 1 Simulation results of the magnetic reconnection separatrix region. $\mathbf{a}, \mathbf{b}$ color contours for $E_{z}$ and local degree of nongyrotropy $\left(D_{\mathrm{ng}}\right)$. The $z$-axis is perpendicular to the current sheet, $x$-axis is along the background magnetic field, and $y$-axis is in the out-of-plane direction to complete the right-handed orthogonal coordinate system. White thick curve lines represent the separatrix field lines. Black curves are in-plane magnetic field lines ( $A_{y}$ contours). $B_{x}$ is positive for $z>0$. Hall field $E_{z}$ and $D_{\text {ng }}$ are enhances in separatrix layers. c Terms in generalized Ohm's law at the intercepts of $x=902 d_{e}$. Red dashed lines denote the 'SUM' of $-\left(\boldsymbol{V}_{i} \times \boldsymbol{B}\right) z_{1}(\boldsymbol{J} \times \boldsymbol{B} /$ ne $) z_{\text {, and }}-\left(\nabla \cdot \boldsymbol{P}_{e} /\right.$ ne $) z$. Black dotted lines plot the $D_{\mathrm{ng}}$. $\mathbf{d}$ The three components of the current density at the left of the $X$-line (not shown). The right exhaust region is open, i.e., no island exists on this side, so the electrons can be freely expelled out of this region. Here, we zoom in to discuss the open exhaust on the right side, but the physics addressed in this paper can be applied to the island side exhaust as well. Figure 1a shows the color contours $E_{z}$ to illustrate the Hall electric field around the separatrix. Figure $1 \mathrm{~b}$ shows another important parameter for both EDR and separatrix, the local degree of electron nongyrotropy $D_{\mathrm{ng}}=2 \sqrt{\sum_{i j} N_{i j}^{2}} / \operatorname{Tr}\left(P_{e}\right)\left[N_{i j}\right.$ is the nongyrotropic elements of the full electron pressure tensor $\boldsymbol{P}_{e}$, see Aunai et al. (2013)]. Two parallel bands of enhanced $E_{z}$ and $D_{\text {ng }}$ outline the EDR centered at the $X$ line (Fig. 1a, b). The electron nongyrotropy in EDR has been discussed by other authors (e.g., Scudder and Daughton 2008; Aunai et al. 2013; Shuster et al. 2015), which is not the major concern in this paper. Both $E_{z}$ and $D_{\text {ng }}$ extend from near the end of the EDR, and diverge away from the $z=0$ plane (neutral plane) roughly along the magnetic separatrix field lines. Later, we will show that the enhancement of $E_{z}$ along separatrix line marks the region where $J \times B$ term dominates, which is defined as the separatrix region in this paper (the detailed definition of the separatrix region are introduced later). Hence, the enhanced $E_{z}$ region can approximately represent the separatrix region in the $2 \mathrm{D}$ contour plot. The magnetic separatrix is inside the separatrix, implying that the Hall effect can extend outside of the magnetic separatrix. In addition, we will show that the enhanced $D_{\mathrm{ng}}$ region along magnetic separatrix line marks the boundary of the accelerated electrons, which is consistent with the previously reported electron separatrix (e.g., Lindstedt et al. 2009).

To show the properties in the separatrix region at downstream of the $X$-line, Fig. 1c, $d$ shows the plot of the terms in Ohm's law and current density, respectively, along the intercept marked as the white vertical line in Fig. 1a, b (as it is roughly symmetric to neutral plane, here we only show the $z>0$ domain to make the picture clear). The intercept is at $x=902 d_{e}$, approximately $3 d_{i}$ away from the $X$-line. In Fig. 1c, black solid curve shows the intercept of $E_{z}$, and $D_{\mathrm{ng}}$ is denoted by black dotted curve. The colored curves represent the $z$-components of the terms in the generalized Ohm's law (electron inertial term is neglected because of their negligible contributions):

$$
\vec{E}+\vec{V} \times \vec{B}=\frac{\vec{J} \times \vec{B}}{\text { ne }}-\frac{\nabla \cdot \overleftrightarrow{P_{e}}}{\text { ne }}
$$

The force balance result shows that the Hall term $\boldsymbol{J} \times \boldsymbol{B} /$ ne is greater than other terms, and is consistent with previous PIC results showing that $E_{z}$ is primarily balanced by the $-\boldsymbol{V}_{\boldsymbol{e}} \times \boldsymbol{B}$ term (combination of $-\boldsymbol{V}_{\boldsymbol{i}} \times \boldsymbol{B}$ 
and the Hall term) across the separatrix within several $d_{i}$ from the $X$-line (Chen et al. 2008). The Hall term becomes stronger than $E z$ at $z=26 d_{e}$ near the inflow region, and has a sudden enhancement at $z=19 d_{e}$ near the exhaust region. We define the region between these two points as the separatrix region in this paper. Here, we further show that the electron pressure tensor contribution through $\nabla \cdot P_{\mathrm{e}} /$ ne is significant in the region of enhanced $D_{\text {ng }}$ and partially counteracts the Hall term. The above two terms together with the $-\boldsymbol{V}_{\boldsymbol{i}} \times \boldsymbol{B}$ term balance $E_{z}$ (see, the red dashed line).

Figure $1 \mathrm{~d}$ shows the three components of the current density. The $x$-component (blue curve) changed its sign near the $D_{\text {ng }}$ peak. On the inflow side, $\mathrm{J}_{x}$ points away from the $X$-line, while it points toward $X$-line at the outflow side, consisting of the Hall current system. The $J_{y}$ component (green curve) also has a peak near the $D_{\mathrm{ng}}$ peak. Its magnitude is nearly one order smaller than the current density at the current center $(z=0)$ in this simulation result. However, it makes the current sheet deviate from the Harris current distribution. Besides, $J_{y}$ component peaks when $J_{x}$ changes its sign, which means the current changed its direction in the separatrix region near the $D_{\text {ng }}$ peak. This analysis suggests that the complex current system in the separatrix has close relation to the electron nongyrotropy, as well as the $\nabla \cdot P_{e} /$ ne term.

To analyze the nongyrotropic feature, Fig. $2 \mathrm{a}-\mathrm{c}$ shows the plots of the eVDFs at $x=902 d_{\mathrm{e}}$. The locations to get the eVDFs are marked in Fig. $1 \mathrm{a}, \mathrm{b}$ by small white rectangles. The eVDFs are organized in $v_{\perp 2}-v_{\perp 1}$ space, the plane perpendicular to the magnetic field, where $\boldsymbol{v}_{\perp 1}$ is in the direction of $\boldsymbol{E} \times \boldsymbol{B}$, and $\boldsymbol{v}_{\perp 2}$ the direction of $\boldsymbol{B} \times \boldsymbol{v}_{\perp \mathbf{1}}$. The eVDFs calculated at the magnetic separatrix are gyrotropic (see Fig. 2a), and the maximum energy is small compared with the eVDFs in the enhanced $D_{\text {ng }}$ region (Fig. 2b) and the exhaust region (Fig. 2c, at exhaust side of the enhanced $D_{\text {ng }}$ region). The eVDF at the enhanced $D_{\text {ng }}$ region (Fig. 2b) contains a gyrotropic cold core (marked by the red circle) similar to that at the magnetic separatrix (Fig. 2a) and a nongyrotropic population with larger perpendicular velocities. To illustrate the changes in the perpendicular energy when crossing the separatrix region, Fig. $2 \mathrm{~d}$ shows the electron spectra within the pitch-angle of $\left[80^{\circ}, 100^{\circ}\right]$ along the intercepts at $x=902$ $d_{e}$ (marked as the vertical white lines in Figs. 1a, b). The spectrum displays that the maximum perpendicular velocities drop sharply at the enhanced $D_{\mathrm{ng}}$ region (marked by red inverted triangles in Fig. 2d). This demonstrates that the enhanced $D_{\mathrm{ng}}$ region marks the boundaries of the heated/accelerated outcoming electrons, i.e., the electron separatrix (the inflow electrons are accelerated/heated parallel but not perpendicular to the magnetic field (see Chen et al. $(2008,2009)$ for evidence from PIC and space observations as well as explanations).

The drastic drop of perpendicular electron populations across the electron separatrix implies a finite gyroradius effect, as evidenced by the expected distribution in Fig. 2e. An electron gyrates around the field line with a gyroradius of $r_{\mathrm{ce}}=m_{e} V_{\perp} / \mathrm{eB}$. Electrons with lower energy and smaller gyroradii (black circles with arrows) exist on both sides of the electron separatrix/boundary (presented as thick purple line), while the accelerated electrons with larger gyroradii (red circles with arrows) exist only below the electron separatrix. Accumulating the electrons in the green box stretched across the boundary, we obtain the nongyrotropic distribution function as shown in the right panel of Fig. 2e. The gray population centered at the origin consists of the low energy electrons, while the pink nongyrotropic population is composed of the accelerated electrons, gyro motions of which are mainly in the $Y$ direction near the electron separatrix. The eVDFs shown in Figs. $2 b$ are consistent with this picture. In Fig. $2 \mathrm{~b}$, the projections of the $-V_{y}$ axis are marked by dashed black lines $\left(v_{\perp 2}=-0.1 v_{\perp 1}\right)$. The nongyrotropic population with velocities larger than $\sim 10 V_{A}$ (see the red cycle in Fig. 2b) is approximately symmetric with respect to the $-V_{y}$ axis, indicating that the nongyrotropic populations are mainly moving in the $-Y$ direction.

To illustrate how the electron separatrix and the finite gyroradius effect impact the current system, we decomposed the current density and bulk velocities in Fig. 3. The vertical solid line marks the $D_{\text {ng }}$ peak, while the two vertical dashed lines mark the separatrix region. The parallel current density (black curve in Fig. 3a) is mainly carried by electrons (red in Fig. 3b) in the inflow side of $D_{\text {ng }}$ peak. In the outflow side, the parallel ion velocity (black curve in Fig. 3b) becomes significant, but is still overridden by electron. The perpendicular current density is mainly in the $y$-direction (green curve in Fig. 3a). In the separatrix region, the electron (red in Fig. 3d) is the major current carrier, while ion (black in Fig. 3d) is the major carrier in the outflow region. In the separatrix region, $E \times B$ drift is the dominant except at the peak $D_{\text {ng }}$ region, where $V_{e \perp, y}$ sharply is enhanced to exceed $V_{E \times B, y}$ On the other hand, the bulk velocity of the coldcore components of the eVDF in Fig. $2 \mathrm{~b}$ agrees well with the local $E \times B$ drift velocities (not shown). Therefore, the nongyrotropic electrons with high perpendicular velocities are the contributors that enable $V_{e y}$ to exceed the $E \times B$ drift velocity. From the viewpoint of fluid, the enhanced $V_{e \perp, y}$ is contributed by the diamagnetic drift. Figure $3 f$ shows the electron diamagnetic drift $V_{e, \text { dia }}=B \times \nabla \cdot P_{e} / \mathrm{neB}^{2}$ (smoothed by means of a window 

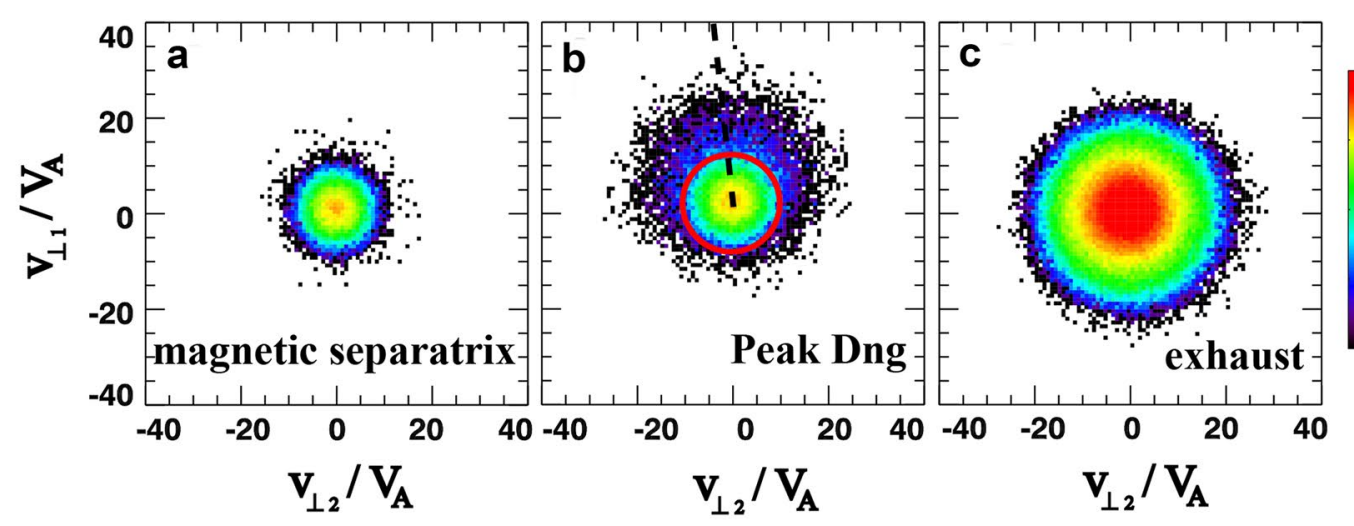

d

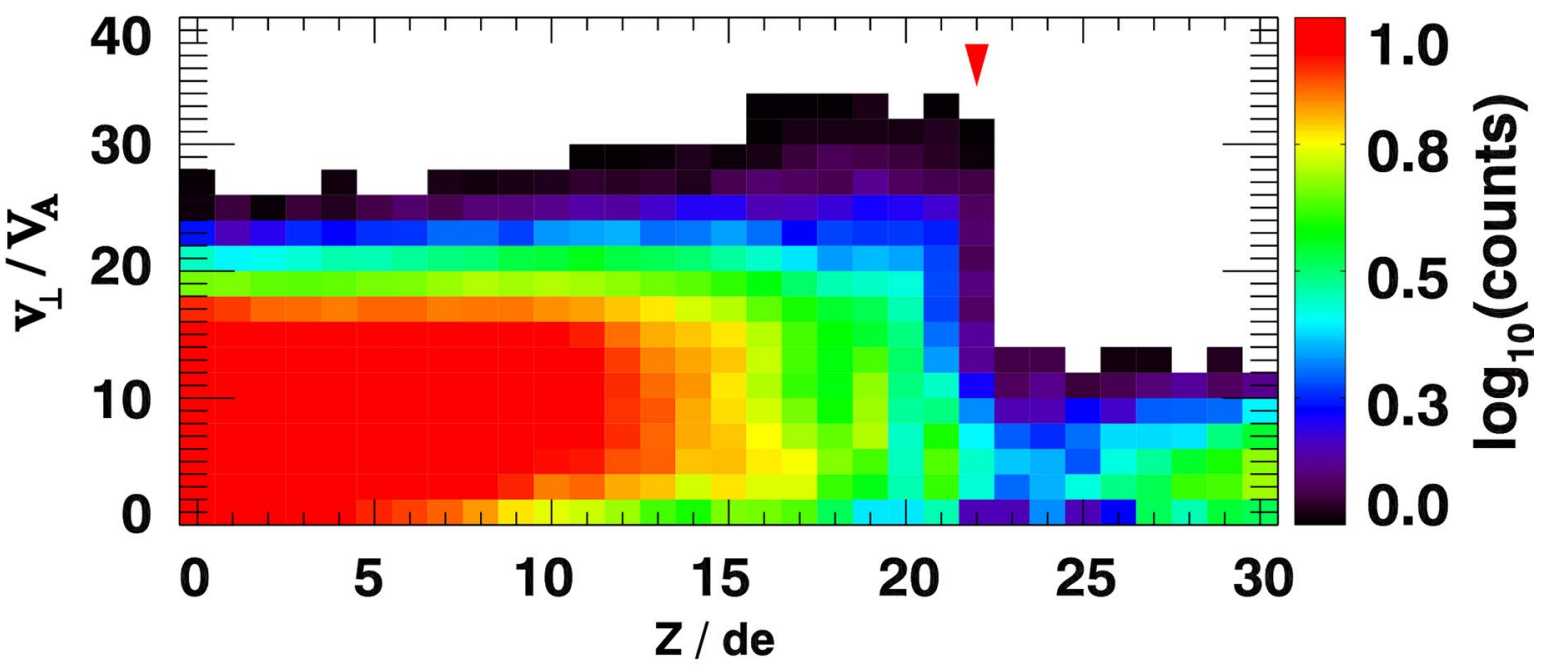

e
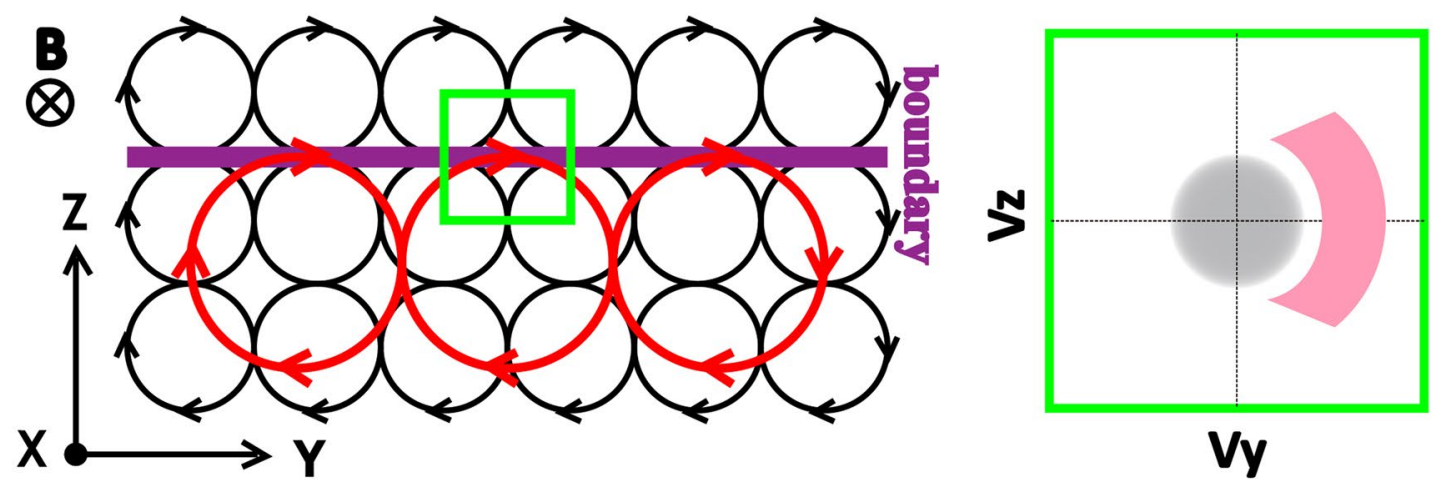

Fig. 2 Electron distributions on the electron separatrix. a-c Electron velocity distribution functions (eVDFs) in the plane perpendicular to the field lines. The locations to get the eVDFs are marked in Fig. $1 \mathrm{a}$, b by small white rectangles. $\mathbf{d}$ electron spectra within the pitch-angles of $\left[80^{\circ}, 100^{\circ}\right]$, calculated at the white vertical line in Fig. 1a. The red inverted triangle marks the position of the peak $D_{\mathrm{ng}}$. The electron spectra show that the maximum perpendicular energy drops sharply at the enhanced $D_{\text {ng }}$ region, implying that the enhanced $D_{\text {ng }}$ region marks the boundaries for the energetic electrons accelerated in the diffusion region. e Sketch map for the generation of nongyrotropy due to the finite gyroradius effect. The black circles with arrows present low-energy electrons. The red circles with arrows present accelerated electrons. The thick purple line is the boundary of the accelerated electrons, which presents the electron separatrix. The right plot is the relevant eVDF calculated on the electron separatrix in the region represented by the green box stretched across the boundary. The gray population in the eVDF is composed of low-energy electrons, while the pink nongyrotropic population is composed of the accelerated electrons 


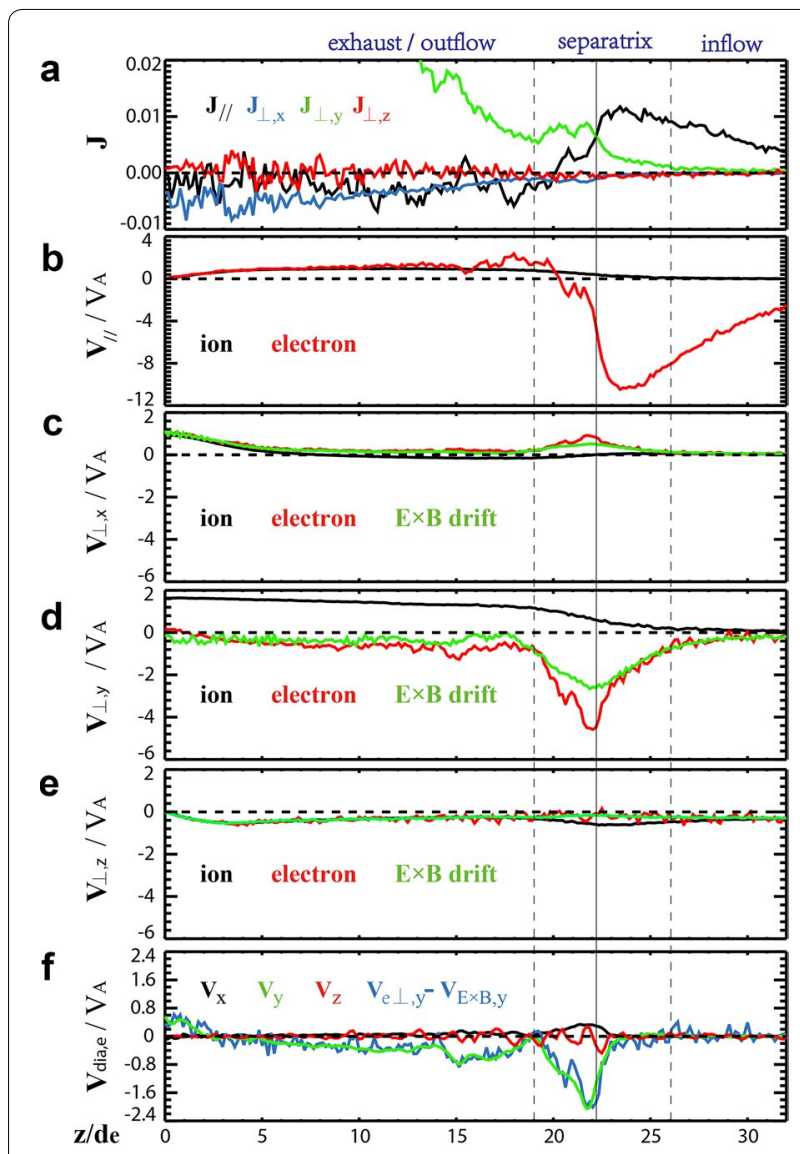

Fig. 3 The decompositions of the current and plasma flows. a The current density. The black curve presents the parallel current density, and blue for $x$-component of the perpendicular current, green for $y$-component of the perpendicular current, red for z-component of the perpendicular current. b The parallel bulk velocity for ions (black) and electrons (red). $\mathbf{c}-\mathbf{e} x-, y-, z$-components of the perpendicular bulk velocity for ions (black), electrons (red), and the $E \times B$ drift velocity (green). f The electron diamagnetic drift velocity (black, green, and red for $x$ - $y$-, $z$-components respectively), and $y$-component of the difference between the perpendicular electron bulk velocity and the $E \times B$ drift, i.e., $\delta V_{e \perp, y}=V_{e \perp, y}-V_{E \times B, y}$ (green). The vertical solid line marks the $D_{\mathrm{ng}}$ peak, while the two vertical dashed lines mark the separatrix region where $E_{z}$ is enhanced

of $\sim 1 d_{\mathrm{e}}$ ) and $\delta V_{e \perp, y}=V_{e \perp, y}-V_{E \times B, y}$ (blue curve). It shows clearly that the diamagnetic drift has a significant effect on the electron separatrix (see, green curve in Fig. 3f). Figure 3e shows that the inflow ion (black) can cross the separatrix region transversely with the speed overriding $E \times B$ drift speed, indicating that the ion is not frozen-in and can be accelerated by the Hall field $E_{z}$, consistent with previous theories. The perpendicular electron velocity has no significant component in the $z$-direction (red in Fig. 3e). $V_{e \perp, x}$ (red in Fig. 3c) is slightly greater than $V_{E \times B, y}$, which is also caused by the diamagnetic drift (black in Fig. 3f).
The current flow changes direction from along the field line to perpendicular to the field line when crossing the separatrix region from inflow to exhaust region. The changing of the current flow is mainly caused by the changing of the electron flow. Figure 4 shows the orientations of the current flow and the electron velocity and other physical parameters when crossing the separatrix. The azimuthal angles (in degree) in Fig. 4a, b (the angle between the $+x$ axis and the velocity vector projected in the xy-plane, calculated in the clockwise orientation) clearly show the sudden changes in of the current and electron flows in the xy-plane, respectively, whereas, there are no obvious jumps in the magnetic field (Fig. 4c) and the number densities (Fig. 4d). The total electron pressure is quickly enhanced at the peak $D_{\text {ng }}$ region (black in Fig. 4e), which is mainly contributed by the parallel electron pressure (green in Fig. 4e). The perpendicular electron pressure (red in Fig. 4e) is increased gradually. The quick enhancement of the parallel electron pressure

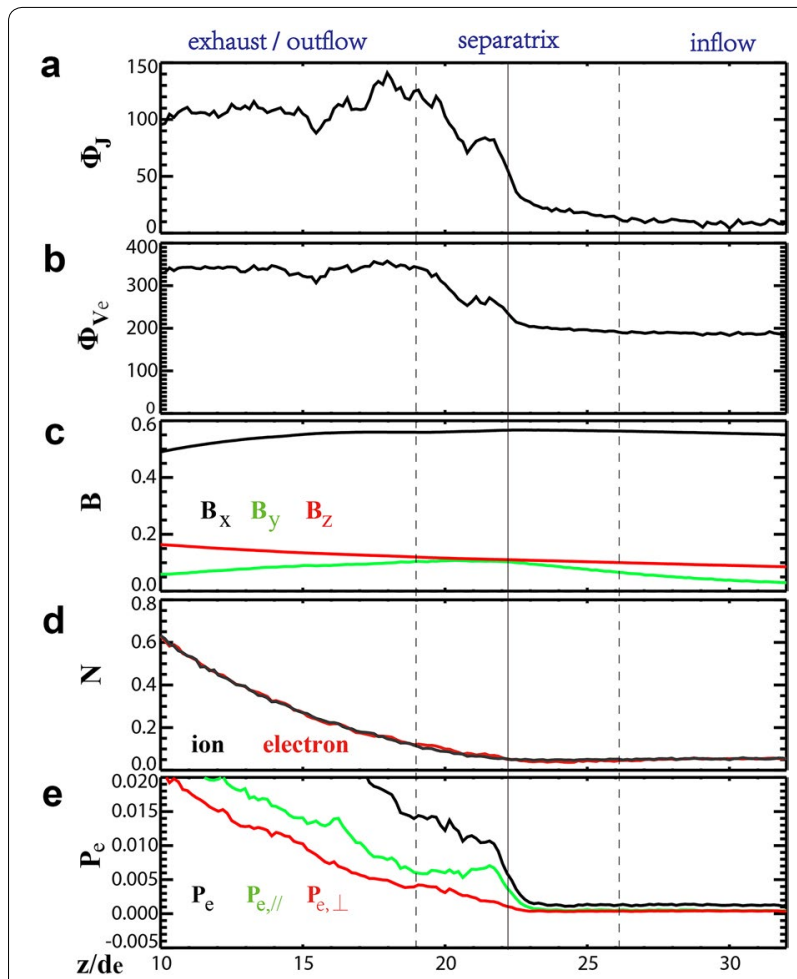

Fig. 4 Jump conditions when crossing the electron separatrix. a The azimuthal angle (in degree) of the current flow. The azimuthal angle is calculated in the xy-plane and ranged from $0^{\circ}$ to $360^{\circ}$. $0^{\circ}$ means the current flows along the $+x$ axis. The angle is counted in the clockwise orientation. $\mathbf{b}$ The azimuthal angle of the electron bulk velocity. c The three components of the magnetic field. $\mathbf{d}$ The number densities of the ion (black) and electron (red). e The total electron pressure (black), the parallel electron pressure (green), and the perpendicular electron pressure (red). The vertical solid line marks the $D_{\text {ng }}$ peak, while the two vertical dashed lines mark the separatrix region where $E_{z}$ is enhanced 
indicates that the electrons can get more parallel heating in the $X$-line and in the exhaust region, rather than in the inflow region. The absence of the jump in the perpendicular electron pressure indicates that the diamagnetic drift and nongyrotropic feature are the secondary effects which cannot make any impact on the pressure balance. From this analysis, it can be suggested that the electron separatrix is a more important boundary than the magnetic separatrix, responsible for the changing of the orientation of current flow, the enhancements of the electron pressure tensor and of the out-of-plane electron velocity, all of which occur at the electron separatrix.

\section{Conclusion}

We have demonstrated that the out-of-plane current density has a peak near the electron separatrix, which implies that a thin current sheet formed in the separatrix region. The thin current sheet is mainly contributed by the electron diamagnetic drift, which is primarily caused by the finite gyroradius effect of outgoing accelerated electrons. Meanwhile, the finite gyroradius effect leads to nongyrotropic electron distributions and enhancement of $D_{\text {ng }}$ along the electron separatrix. The nongyrotropic electrons contribute to the perpendicular velocity $V_{\text {ey }}$, and make $V_{\text {ey }}$ exceed the $E \times B$ drift velocity, enhancing the Hall effect. The electron separatrix is marked by the enhanced out-of-plane electron velocity, electron nongyrotropy, and divergence of electron pressure tensor, while it has no obvious effect on magnetic field and number density. In addition, the current and electron flows changed their orientations at the electron separatrix. All these parameters can be the candidates to identify the electron separatrix and the separatrix region.

The time stage in this study is chosen to be near the reconnection peak. It is at an early stage in the whole reconnection evolution process, to get rid of turbulentlike features which arise at the later simulation stage. The original particles have not been expelled from the near $X$-line region at the early stage, so the high plasma density leads to a peak current density at the neutral center. However, the density decreased at later simulation stage when the dense plasma is expelled out by the reconnection outflow. Then, the current density at the neutral center is reduced to less than that on separatrix region (not shown). That is to say the thin current layer studied in this paper can evolve into the bifurcated current sheet which has been observed in the magnetotail (Runov et al. 2003, 2006).

The thin current sheet and the associated finite gyroradius effect in the separatrix region discussed in this paper are different from those in the previous investigations of EDR on magnetopause (e.g., Hesse et al. 2014; Burch et al. 2016; Shay et al. 2016). In EDR on magnetopause, the electrons are accelerated by the in-plane electric field during their meandering motions around the neutral line, in turn leading to the enhanced electron nongyrotropy and forming the crescent-shaped electron distribution on the magnetosphere side of the EDR (Shay et al. 2016). In contrast, in the separatrix region, the magnetic fields are not reversed in the thin current sheet, and the accelerated electrons make cyclotron motions rather the meandering motions. In addition, the accelerated electrons had already been accelerated before arriving at the separatrix region. The boundary for the accelerated electrons in separatrix region is maintained by the magnetic reconnection process. In other words, it is the reconnection process that generated the electron boundary to form the bifurcated thin current sheet in the separatrix region apart from the neutral line. Hence, the bifurcated current sheets can be a signature of active reconnection region, similar to the hypothesis proposed by Thompson et al. (2006). The phenomena discussed here could be independent of the background plasma environment, so we can suggest that the thin current sheet seen on the separatrix region could also be seen in the reconnection region on magnetopause. Furthermore, the findings from this study would also be helpful to diagnose the reconnection region in the magnetotail.

\section{Abbreviations \\ PIC: Particle-in-cell; EDR: electron diffusion region; eVDFs: electron velocity distribution functions.}

\section{Authors' contributions}

RG analyzed the data and drafted the paper. Prof. ZP and YW helped to discuss and organize the details of the paper. All authors read and approved the final manuscript

\section{Author details}

${ }^{1}$ Key Laboratory of Earth and Planetary Physics, Institute of Geology and Geophysics, Chinese Academy of Sciences, Beijing 100029, China. ${ }^{2}$ School of Earth and Space Sciences, Peking University, Beijing, China.

\section{Acknowledgements}

Ruilong Guo was supported by the China Scholarship Council for 1-year study at University of New Hampshire. Ruilong Guo thanks William Daughton and $\mathrm{Li}$-Jen Chen for providing the simulation data, and thanks Li-Jen Chen, Shan Wang, Jason Shuster, Guanlai Li, and Roy Tobert for their help and fruitful discussion when analyzing the simulation data when studying in UNH.

\section{Competing interests}

The authors declare that they have no competing interests.

\section{Availability of data and materials}

The simulation data are provided by William Daughton and Li-Jen Chen when Ruilong Guo studying in University of New Hampshire. The simulation data are available upon request from the authors.

\section{Consent for publication}

Not applicable.

Ethics approval and consent to participate

Not applicable. 


\section{Funding}

The work was supported by the National Science Foundation of China $(41525016,41474155,41661164034)$. Y. Wei was supported by Thousand Young Talents Program of China.

\section{Publisher's Note}

Springer Nature remains neutral with regard to jurisdictional claims in published maps and institutional affiliations.

Received: 31 December 2016 Accepted: 29 August 2017

Published online: 06 September 2017

\section{References}

Aunai N et al (2013) Electron nongyrotropy in the context of collisionless magnetic reconnection. Phys Plasmas 20:092903. doi:10.1063/1.4820953

Burch JL, Phan TD (2016) Magnetic reconnection at the dayside magnetopause: advances with MMS. Geophys Res Lett 43(16):8327-8338. doi:10.1 002/2016GL069787

Burch JL et al (2016) Electron-scale measurements of magnetic reconnection in space. Science. doi:10.1126/science.aaf2939

Cattell C et al (2005) Cluster observations of electron holes in association with magnetotail reconnection and comparison to simulations. J Geophys Res 110:A01211. doi:10.1029/2004JA010519

Chen LJ et al (2008) Evidence of an extended electron current sheet and its neighboring magnetic island during magnetotail reconnection. J Geophys Res 113:A12213. doi:10.1029/2008JA013385

Chen LJ et al (2009) Multispacecraft observations of the electron current sheet neighboring magnetic islands, and electron acceleration during magnetotail reconnection. Phys Plasmas 16:056501. doi:10.1063/1.3112744

Chen LJ, Hesse M, Wang S, Bessho N, Daughton W (2016) Electron energization and structure of the diffusion region during asymmetric reconnection. Geophys Res Lett 43(6):2405-2412. doi:10.1002/2016GL068243

Daughton W et al (2006) Fully kinetic simulations of undriven magnetic reconnection with open boundary conditions. Phys Plasmas 13:072101. doi:10.1063/1.2218817

Daughton W et al (2009) Influence of Coulomb collisions on the structure of reconnection layers. Phys Plasmas 16:072117. doi:10.1063/1.3191718

Drake JF et al (2003) Formation of electron holes and particle energization during magnetic reconnection. Science 299:873-877

Drake JF, Shay MA, Swisdak M (2008) The Hall fields and fast magnetic reconnection. Phys Plasmas 15:042306. doi:10.1063/1.2901194

Egedal J, Fox W, Katz N, Porkolab M, Øieroset M, Lin RP, Daughton W, Drake JF (2008) Evidence and theory for trapped electrons in guide field magnetotail reconnection. J Geophys Res 113:A12207. doi:10.1029/2008JA013520

Egedal J, Lê A, Katz N, Chen LJ, Lefebvre B, Daughton W, Fazakerley A (2010) Cluster observations of bidirectional beams caused by electron trapping during antiparallel reconnection. J Geophys Res 115:A03214. doi:10.1029 /2009JA014650

Egedal J, Daughton W, Lê A (2012) Large-scale electron acceleration by parallel electric fields during magnetic reconnection. Nat Phys 8:321-324. doi:10.1038/NPHYS2249

Farrell WM et al (2002) The dominance of electron plasma waves near a reconnection X-line region. Geophys Res Lett 29:1902. doi:10.1029/200 2GL014662

Fujimoto K (2006) Time evolution of the electron diffusion region and the reconnection rate in fully kinetic and large system. Phys Plasmas 13:072904. doi:10.1063/1.2220534

Fujimoto K (2014) Wave activities in separatrix regions of magnetic reconnection. Geophys Res Lett 41:2721-2728. doi:10.1002/2014GL059893

Hesse M, Aunai N, Sibeck D, Birn J (2014) On the electron diffusion region in planar, asymmetric, systems. Geophys Res Lett 41:8673-8680. doi:10.100 2/2014GL061586
Hesse M, Liu YH, Chen L, Bessho N, Kuznetsova M, Birn J, Burch JL (2016) On the electron diffusion region in asymmetric reconnection with a guide magnetic field. Geophys Res Lett 43(6):2359-2364. doi:10.1002/201 6GL068373

Hwang KJ, Goldstein ML, Wendel DE, Fazakerley AN, Gurgiolo C (2013) Cluster observations near reconnection X lines in Earth's magnetotail current sheet. J Geophys Res Space Phys 118:4199-4209. doi:10.1002/jgra.50403

Hwang K-J et al (2016) The substructure of a flux transfer event observed by the MMS spacecraft. Geophys Res Lett 43:9434-9443. doi:10.1002/201 6GL070934

Khotyaintsev YV et al (2006) Formation of inner structure of a reconnection separatrix region. Phys Rev Lett 97:205003. doi:10.1103/ PhysRevLett.97.205003

Lindstedt T et al (2009) Separatrix regions of magnetic reconnection at the magnetopause. Ann Geophys 27:4039-4056

Nakamura T, Nakamura R, Haseagwa H (2016) Spatial dimensions of the electron diffusion region in anti-parallel magnetic reconnection. Ann Geophys 34:357-367. doi:10.5194/angeo-34-357-2016

Norgren C et al (2016) Finite gyroradius effects in the electron outflow of asymmetric magnetic reconnection. Geophys Res Lett 43:6724-6733. doi :10.1002/2016GL069205

Phan TD et al (2016) MMS observations of electron-scale filamentary currents in the reconnection exhaust and near the $X$ line. Geophys Res Lett 43:6060-6069. doi:10.1002/2016GL069212

Retinò A et al (2006) Structure of the separatrix region close to a magnetic reconnection X-line: cluster observations. Geophys Res Lett 33:L06101. doi:10.1029/2005GL024650

Runov A et al (2003) Current sheet structure near magnetic X-line observed by cluster. Geophys Res Lett 30(11):1579. doi:10.1029/2002GL016730

Runov A et al (2006) Local structure of the magnetotail current sheet: 2001 Cluster observations. Ann Geophys 24:247-262

Scudder J, Daughton W (2008) "Illuminating" electron diffusion regions of collisionless magnetic reconnection using electron agyrotropy. J Geophys Res 113:A06222. doi:10.1029/2008JA013035

Shay MA, Phan TD, Haggerty CC, Fujimoto M, Drake JF, Malakit K, Cassak PA, Swisdak M (2016) Kinetic signatures of the region surrounding the $X$ line in asymmetric (magnetopause) reconnection. Geophys Res Lett 43:4145-4154. doi:10.1002/2016GL069034

Shuster JR et al (2015) Spatiotemporal evolution of electron characteristics in the electron diffusion region of magnetic reconnection: Implications for acceleration and heating. Geophys Res Lett. doi:10.1002/2015GL063601

Swisdak M (2016) Quantifying gyrotropy in magnetic reconnection. Geophys Res Lett 43:43-49. doi:10.1002/2015GL066980

Thompson SM, Kivelson MG, El-Alaoui M, Balogh A, Reme H, Reme LM (2006) Bifurcated current sheets: statistics from Cluster magnetometer measurements. J Geophys Res 111:A03212. doi:10.1029/2005JA011009

Torbert RB et al (2016) Estimates of terms in Ohm's law during an encounter with an electron diffusion region. Geophys Res Lett 43:5918-5925. doi:10.1002/2016GL069553

Viberg $\mathrm{H}$ et al (2013) Mapping HF waves in the reconnection diffusion region. Geophys Res Lett 40:1032-1037. doi:10.1002/grl.50227

Wang S, Chen LJ, Bessho N, Kistler LM, Shuster JR, Guo R (2016) Electron heating in the exhaust of magnetic reconnection with negligible guide field. J Geophys Res Space Phys 121:2104-2130. doi:10.1002/2015JA021892

Yao Z et al (2013) Current structures associated with dipolarization fronts. Geophys Res Space Phys 118:6980-6985. doi:10.1002/2013JA019290

Yao Z et al (2016) Substructures within a dipolarization front revealed by high-temporal resolution Cluster observations. J Geophys Res Space Phys 121:5185-5202. doi:10.1002/2015JA022238

Yao Z et al (2017) A direct examination of the dynamics of depolarization fronts using MMS. J Geophys Res Space Phys 122:4335-4347. doi:10.100 2/2016JA023401 